International Journal of Pure and Applied Mathematics

Volume 96 No. 1 2014, 27-35

ISSN: 1311-8080 (printed version); ISSN: 1314-3395 (on-line version)

url: http://www.ijpam.eu

doi: http://dx.doi.org/10.12732/ijpam.v96i1.3

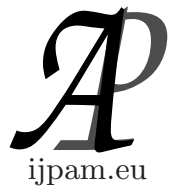

\title{
ABOUT ANALYSIS AND MODELING OF THE OPEN MESSAGE SWITCHING SYSTEM
}

\author{
Saulius Minkevičius ${ }^{1}$, Vladimiras Dolgopolovas ${ }^{2} \S$ \\ ${ }^{1,2} \mathrm{VU}$ Institute of Mathematics and Informatics \\ Akademijos 4, LT-08663 Vilnius, LITHUANIA \\ ${ }^{1,2}$ Vilnius University, Naugarduko 24 \\ LT-03225 Vilnius, LITHUANIA
}

\begin{abstract}
The modern queueing theory is one of the powerful tools for a quantitative and qualitative analysis of communication systems, computer networks, transportation systems, and many other technical systems. The paper is designated to the analysis of queueing systems, arising in the networks theory and communications theory (called open queueing network). The authors of this research in the sphere of queueing theory present the theorem about the law of the iterated logarithm (LIL) for the queue length in open queueing network and its application to the mathematical model of the open message switching system.
\end{abstract}

AMS Subject Classification: 60K25, 60G70, 60F17

Key Words: models of information system, open message switching system, open queueing network, queue length, heavy traffic, a law of the iterated logarithm

\section{Problem Formulation}

At first the authors continues the research in queueing theory about LIL in queueing systems and presents theorem for the queue length of a customers in open queueing network. We note that papers on the queue length in heavy

Received: March 16, 2014

(C) 2014 Academic Publications, Ltd.

${ }^{\S}$ Correspondence author url: www.acadpubl.eu 
traffic for a multiphase queue and open queueing network are sparse. In $[6,7]$ tandem queues with blocking in heavy traffic were investigated. In [9], functional limit theorems for the queue length of customers in multiphase queues in various conditions of heavy traffic are proved. In [3] some results on the queue length in multiphase queues were surveyed. In [9] laws of the iterated logarithm for a total queue length of customers and a queue length of customers in multiphase queues were presented. In [10], functional limit theorems on complex transient phenomena for a queue length in multiphase queues are proved. In [4,5] diffusion approximations for a two-node tandem queue with general renewal process input and independent exponential service times at each node were presented.

Note that the research of LIL in more general systems than the queueing system $G I / G / 1$ or multiphase queueing systems and open queueing network has just started (see [1]). In [8,9], LIL is proved in heavy traffic for the queue length of customers, waiting time of a customer, a virtual waiting time of a customer in a multiphase queueing system. In [11], also gives the proof of the theorem on LIL under the conditions of heavy traffic for a virtual waiting time of a customer in the open Jackson network.

In this paper, we investigated an open queueing network model in heavy traffic. The LIL for the queue length of customers in an open queueing network has been presented. The main tool for the analysis of these queueing systems in heavy traffic is a functional LIL for renewal process (the proof can be found in [12] and [2]).

The service discipline is "first come, first served" (FCFS). We consider open queueing networks with the FCFS service discipline at each station and general distributions of interarrival and service times. The queueing network we studied has $k$ single server stations, each of which has an associated infinite capacity waiting room. Every station has an arrival stream from outside the network, and the arrival streams are assumed to be mutually independent renewal processes. Customers are served in the order of arrival and after service they are randomly routed to either another station in the network, or out of the network entirely. Service times and routing decisions form mutually independent sequences of independent identically distributed random variables.

The basic components of the queueing network are arrival processes, service processes, and routing processes. In particular, there are mutually independent sequences of independent identically distributed random variables $\left\{z_{n}^{(j)}, n \geq 1\right\},\left\{S_{n}^{(j)}, n \geq 1\right\}$ and $\left\{\Phi_{n}^{(j)}, n \geq 1\right\}$ for $j=1,2, \ldots, k$; defined on the probability space. Random variables $z_{n}^{(j)}$ and $S_{n}^{(j)}$ are strictly positive, and 
$\Phi_{n}^{(j)}$ have support in $\{0,1,2, \ldots, k\}$. We define $\mu_{j}=\left(M\left[S_{n}^{(j)}\right]\right)^{-1}>0, \sigma_{j}=$ $D\left(S_{n}^{(j)}\right)>0$ and $\lambda_{j}=\left(M\left[z_{n}^{(j)}\right]\right)^{-1}>0, a_{j}=D\left(z_{n}^{(j)}\right)>0, j=1,2, \ldots, k$; with all of these terms assumed finite. Denote $p_{i j}=P\left(\Phi_{n}^{(i)}=j\right)>0, j=$ $1,2, \ldots, k$. In the context of the queueing network, the random variables $z_{n}^{(j)}$ function as interarrival times (from outside the network) at the station $j$, while $S_{n}^{(j)}$ is the $n$th service time at the station $j$, and $\Phi_{n}^{(j)}$ is a routing indicator for the $n$th customer served at the station $j$. If $\Phi_{n}^{(i)}=j$ (which occurs with probability $p_{i j}$ ), then the $n$th customer served at the station $i$ is routed to the station $j$. When $\Phi_{n}^{(i)}=0$, the associated customer leaves the network. The matrix $\mathrm{P}$ is called a routing matrix.

First let us define $Q_{j}(t)$ as the queue length of customers at the $j$ th station of the queueing network in time $t$;

$$
\begin{gathered}
\hat{\sigma}_{j}^{2}=\left(\lambda_{j}\right)^{3} \cdot D z_{n}^{(j)}+\sum_{i=1}^{k}\left(\mu_{i}\right)^{3} \cdot D S_{n}^{(i)} \cdot\left(p_{i j}\right)^{2}+\left(\mu_{j}\right)^{3} \cdot D S_{n}^{(j)}>0, \\
\hat{\beta}_{j}=\lambda_{j}+\sum_{i=1}^{k} \mu_{i} \cdot p_{i j}-\mu_{j}>0, j=1,2, \ldots, k .
\end{gathered}
$$

We assume that the following condition is fulfilled:

$$
\lambda_{j}+\sum_{i=1}^{k} \mu_{i} \cdot p_{i j}>\mu_{j}, j=1,2, \ldots, k .
$$

Note that this condition guarantees that, with probability one there exists a queue length of customers and this queue length of customers is constantly growing.

\section{Main Result}

One of the results of the paper is a theorem on LIL for the queue length of customers in an open queueing network.

Theorem 2.1. If conditions (1) are fulfilled, then

$$
P\left(\varlimsup_{t \rightarrow \infty} \frac{Q_{j}(t)-\hat{\beta}_{j} \cdot t}{\hat{\sigma}_{j} \cdot a(t)}=1\right)=P\left(\varliminf_{t \rightarrow \infty} \frac{Q_{j}(t)-\hat{\beta}_{j} \cdot t}{\hat{\sigma}_{j} \cdot a(t)}=-1\right)=1,
$$


$j=1,2, \ldots, k$ and $a(t)=\sqrt{2 t \ln \ln t}$.

Proof. The proof of theorem is connected with the proof of Theorem 3.1 from paper [11], and we omit this. The proof of Theorem 2.1 is complete.

\section{On the Model of the Open Message Switching Facility}

In this part of the paper, we will present an application of the proved theorem - a mathematical model of open message switching system.

As noted in the introduction, open network queueing systems are of special interest both in theory and in practical applications. Such systems consist of several service nodes, and each arriving customer is served in the order of arrival and after service they are randomly routed to either another station in the network, or out of the network entirely. A typical example is provided by queueing systems with identical service. Such systems are very important in applications, especially to open message switching systems. In fact, in many communication systems the transmission times of the customers do not vary in the delivery process.

So, we investigate a message switching system which consists of $k$ service nodes and in which $S_{n}^{(j)}=S_{n}, j=1,2, \ldots, k$ (the service process is identical in nodes of the system).

Also, let us note

$$
\begin{gathered}
\mu=\left(M\left[S_{n}\right]\right)^{-1}>0 \\
\tilde{\sigma}_{j}^{2}=\left(\lambda_{j}\right)^{3} \cdot D z_{n}^{(j)}+(\mu)^{3} \cdot D S_{n} \cdot\left(1+\sum_{i=1}^{k}\left(p_{i j}\right)^{2}\right), \\
\beta_{j}=\lambda_{j}-\mu \cdot\left(1-\sum_{i=1}^{k} p_{i j}\right)>0, j=1,2, \ldots, k .
\end{gathered}
$$

We assume that the following conditions are fulfilled:

$$
\beta_{j}>0, j=1,2, \ldots, k \text {. }
$$

Similarly as in the proof of Theorem 2.1, we present the following theorem and corollary on LIL for the queue length of messages in open message switching systems. 
Theorem 3.1. If conditions (2) are fulfilled, then

$$
P\left(\varlimsup_{t \rightarrow \infty} \frac{Q_{j}(t)-\beta_{j} \cdot t}{\tilde{\sigma}_{j} \cdot a(t)}=1\right)=P\left(\varliminf_{t \rightarrow \infty} \frac{Q_{j}(t)-\beta_{j} \cdot t}{\tilde{\sigma}_{j} \cdot a(t)}=-1\right)=1,
$$

$j=1,2, \ldots, k$ and $a(t)=\sqrt{2 t \ln \ln t}$.

Corollary 3.2. If conditions (2) are fulfilled, then for fixed $\varepsilon>0$ there exists $t(\varepsilon)$ such that for every $t \geq t(\varepsilon)$,

$$
(1-\varepsilon) \cdot \tilde{\sigma}_{j} \cdot a(t)+\beta_{j} \cdot t \leq Q_{j}(t) \leq(1+\varepsilon) \cdot \tilde{\sigma}_{j} \cdot a(t)+\beta_{j} \cdot t, \quad j=1,2, \ldots, k,
$$

with probability one.

\section{On the Model of the Multistage Message Switching Facility}

Finally we present investigation of seperate case of Theorem 3.1 (case of multistage message switching system). We investigate here a $k$-phase queue (i.e., after a customer has been served in the $j$-th phase of the queue, he is routed to the $j+1$-th phase of the queue, and, after the service in the $k$-th phase of the queue, he leaves the queue). Let us denote by $t_{n}$ the time of arrival of the $n$-th customer; by $S_{n}^{(j)}$ - the service time of the $n$-th customer in the $j$-th phase; $z_{n}=t_{n+1}-t_{n} ; j=1,2, \cdots, k$.

So, we investigate a multistage message switching system which consists of $k$ service nodes and in which $S_{n}^{(j)}=S_{n}, j=1,2, \ldots, k$ (the service process is identical in phases of the system).

Next, denote $Q_{j}(t)$ as the queue length of messages in $j$-th phase of multistage message switching system at time moment $t ; v_{j}(t)=\sum_{i=1}^{j} Q_{i}(t)$ stands the total queue length of messages until $j$-phase of the multistage message switching system at time moment $t, j=1,2, \ldots, k$ and $t>0$.

Let us define $\beta_{1}=\left(M S_{n}\right)^{-1}, \beta_{0}=\left(M z_{n}\right)^{-1}, \alpha=\beta_{0}-\beta_{1}, \hat{\sigma}_{1}^{2}=D S_{n}$. $\left(M S_{n}\right)^{-3}>0, \hat{\sigma}_{0}^{2}=D z_{n} \cdot\left(M z_{n}\right)^{-3}>0, \quad \tilde{\sigma}^{2}=\hat{\sigma}_{1}^{2}+\hat{\sigma}_{0}^{2}$.

We assume that the following condition is fulfilled:

$$
\alpha>0 .
$$

In [9] is presented theorem and corollary about total queue length of messages in multistage message switching system. 
Theorem 4.1. If condition (3) is fulfilled, then

$$
P\left(\varlimsup_{t \rightarrow \infty} \frac{v_{j}(t)-\alpha \cdot t}{\tilde{\sigma} \cdot a(t)}=1\right)=P\left(\varliminf_{t \rightarrow \infty} \frac{v_{j}(t)-\alpha \cdot t}{\tilde{\sigma} \cdot a(t)}=-1\right)=1
$$

$j=1,2, \ldots, k$ and $a(t)=\sqrt{2 t \ln \ln t}$.

Corollary 4.1. If condition (3) is fulfilled, then for fixed $\varepsilon>$ there exists $t(\varepsilon)$ such that for every $t \geq t(\varepsilon)$,

$$
(1-\varepsilon) \cdot \tilde{\sigma} \cdot a(t)+\alpha \cdot t \leq v_{j}(t) \leq(1+\varepsilon) \cdot \tilde{\sigma} \cdot a(t)+\alpha \cdot t, j=1,2, \ldots, k
$$

with probability one.

We see that the queue length of waiting messages in the first phase gives a major contribution to the queue length of messages for the whole system (see, for example, [3], p. 71).

\section{Computing Example}

We see that Theorem 4.1 implies that for fixed $\varepsilon>0$ there exists $t(\varepsilon)$ such that for every $t \geq t(\varepsilon)$,

$$
(1-\varepsilon) \cdot \tilde{\sigma} \cdot a(t)+\alpha \cdot t \leq v_{j}(t) \leq(1+\varepsilon) \cdot \tilde{\sigma} \cdot a(t)+\alpha \cdot t,
$$

where $a(t)=\sqrt{2 t \ln \ln t}, \beta_{1}=\left(M S_{n}\right)^{-1}, \beta_{0}=\left(M z_{n}\right)^{-1}, \alpha=\beta_{0}-\beta_{1}>0, \hat{\sigma}_{1}^{2}=$ $D S_{n} \cdot\left(M S_{n}\right)^{-3}>0, \hat{\sigma}_{0}^{2}=D z_{n} \cdot\left(M z_{n}\right)^{-3}>0, \quad \tilde{\sigma}^{2}=\hat{\sigma}_{1}^{2}+\hat{\sigma}_{0}^{2}, \quad j=1,2, \ldots, k$.

From this we can obtain

$$
\begin{aligned}
& (1-\varepsilon) \cdot \tilde{\sigma} \cdot a(t)+\alpha \cdot t \leq v_{j}(t) \leq(1+\varepsilon) \cdot \tilde{\sigma} \cdot a(t)+\alpha \cdot t, \\
& \left|M\left(v_{j}(t)-\alpha \cdot t\right)-\{(1-\varepsilon) \cdot \tilde{\sigma} \cdot a(t)\}\right| \leq 2 \cdot \varepsilon \cdot \tilde{\sigma} \cdot a(t) \\
& \left|M\left(\frac{\left.v_{j}(t)-\alpha \cdot t\right)}{\tilde{\sigma} \cdot a(t)}\right)-(1+\varepsilon)\right| \leq 2 \cdot \varepsilon, j=1,2, \ldots, k
\end{aligned}
$$

So from (5) we can get

$$
M v_{j}(t) \sim \alpha \cdot t+(1+\varepsilon) \cdot \tilde{\sigma} \cdot a(t), j=1,2, \ldots, k .
$$

$M v_{j}(t)$ is average total queue length of messages until $j$-th phase of the multistage message switching system at time moment $t, j=1,2, \ldots, k$ and $t>0$. 
We see from (6) that $M v_{j}(t)$ consists of linear function and nonlinear slowly increasing function $(1+\varepsilon) \cdot \tilde{\sigma} \cdot a(t), j=1,2, \ldots, k$.

Now we present a technical example from the computer network practice. Assume that messages arrive at the computer $v_{1}$ at the rate $\lambda$ of 21 per hour during business hours. These messages are served at a rate $\mu$ of 20 per hour in the computer $v_{1}$. After service in the computer $v_{1}$ messages arrive at the second computer $v_{2}$. Also we note that messages are served at a rate $\mu$ of 20 per hour in the computer $v_{2}$. So, messages is served in computers $v_{1}, v_{2}, \ldots, v_{k}$, and after messages are served in computer $v_{k}$, they leave computer network.

So,

$$
\begin{gathered}
\beta_{1}=\left(M S_{n}\right)^{-1}=\mu=20, \quad \beta_{0}=\left(M z_{n}\right)^{-1}=\lambda=21, \quad \alpha=21-20=1, \\
D S_{n}=1 / \mu=1 / 20, D z_{n}=1 / \mu=1 / 21, \\
\hat{\sigma}_{1}^{2}=D S_{n} \cdot\left(M S_{n}\right)^{-3}=(1 / \mu)^{2}=0.0025, \\
\hat{\sigma}_{0}^{2}=D S_{n} \cdot\left(M S_{n}\right)^{-3}=(1 / \mu)^{2}=0.0023, \\
\tilde{\sigma}^{2} \sim 0.0047, \tilde{\sigma} \sim 0.0685, \varepsilon=0.001, t \geq 100 .
\end{gathered}
$$

Thus,

$$
M v_{j}(t) \sim \alpha \cdot t+(1+\varepsilon) \cdot \tilde{\sigma} \cdot a(t)=(1.00) \cdot t+(0.0685) \cdot a(t), j=1,2, \ldots, k .
$$

From (7) we get

$$
\frac{M v_{j}(t)}{t}=(1.00)+(0.0685) \cdot \sqrt{\frac{2 \ln \ln t}{t}}, j=1,2, \ldots, k .
$$

Now we present figure for $\frac{M v_{j}(t)}{t}, j=1,2, \ldots, k$, when $100 \leq t \leq 1000, \varepsilon=$ 0.001 (see (8) and Table 1).

We see that when $\alpha=0.01>0$, average total queue length of messages is about 1 in all message system.

Corollary 5.1. Average total queue length of messages system direcly depends of traffic coefficient $\alpha$ and time $t$ and is the same in all phases of multistage message system. 


\begin{tabular}{|r|c|}
\hline Time $t$ & $\frac{M v_{j}(t)}{t}, j=1,2, \ldots, k$ \\
\hline 100 & 1.0120 \\
200 & 1.0088 \\
300 & 1.0074 \\
400 & 1.0065 \\
500 & 1.0059 \\
600 & 1.0054 \\
700 & 1.0050 \\
800 & 1.0047 \\
900 & 1.0045 \\
1000 & 1.0043 \\
\hline
\end{tabular}

Table 1: Summary of computing results

\section{Acknowledgments}

Research supported in part by the National Complex Programme "Theoretical and Engineering aspects of e-service technology creation and application in high-performing calculation platforms".

\section{References}

[1] Bingham N. H. (1986). Variants of the law of the iterated logarithm. Bulletin London Mathematical Society, 18, 433-467, doi: 10.1112/blms/18.5.433

[2] Iglehart D.L. (1971). Multiple channel queues in heavy traffic. IV. Law of the iterated logarithm. Zeitschrift für Wahrscheinlicht-Keitstheorie und Verwandte Gebiete, 17, 168-180, doi: 10.1007/BF00538869

[3] Karpelevich F.I., Kreinin A.I. (1994). Heavy traffic limits for multiphase queues. American Mathematical Society, Providence.

[4] Knessl C., Tier C. (1999a). Two tandem queues with general renewal input. I and II. SIAM J. Appl. Math., 59(6), 1917-1959, 1960-1997 (electronic).

[5] Knessl C., Tier C. (1999b). A diffusion model for two tandem queues with general renewal input. Comm. Statist. Stochastic Models, 15(2), 299-343, doi: 10.1080/15326349908807538. 
[6] Kogan Ya., Pukhalskii, A. (1985). On tandem queues with blocking in heavy traffic. Performance'84 (Paris, 1984), North-Holland, Amsterdam, 549-558.

[7] Kogan Ya., Pukhalskii, A. (1988). Tandem queue with finite intermediate waiting room and blocking in heavy traffic. Problems Control Inform. Theory, 17(1), 3-13 (in Russian).

[8] Minkevičius S. (1995). On the law of the iterated logarithm in multiphase queueing systems, Lithuanian Mathematical Journal, 35, 360-366.

[9] Minkevčius S. (1997a). On the law of the iterated logarithm in multiphase queueing systems. II, Informatica, 8, 367-376.

[10] Minkevičius S. (1997b). Complex transient processes in multiphase queueing systems. Lietuvos Matematikos Rinkinys, 37(4), 519-531.

[11] Sakalauskas L. L., Minkevičius S. (2000). On the law of the iterated logarithm in open queueing networks. European Journal of Operational Research, 120, 632-640, doi: 10.1016/S0377-2217(99)00003-X.

[12] Strassen V. (1964). An invariance principle for the law of the iterated logarithm. Zeitschrift für Wahrscheinlichkeitstheorie und Verwandte Gebiete, 3, 211-226, doi: 10.1007/BF00534910. 
